\title{
Berardino Palumbo, Piegare i santi. Inchini rituali e pratiche mafiose
}

Marietti, Bologna, 2020

Ferdinando Fava

\section{(2) OpenEdition}

\section{Journals}

Edizione digitale

URL: http://journals.openedition.org/aam/3132

DOI: $10.4000 /$ aam.3132

ISSN: 2038-3215

Editore

Dipartimento Culture e Società - Università di Palermo

Notizia bibliografica digitale

Ferdinando Fava, «Berardino Palumbo, Piegare i santi. Inchini rituali e pratiche mafiose», Archivio

antropologico mediterraneo [Online], Anno XXIII, n. 22 (1) | 2020, online dal 20 juin 2020, consultato il 27 janvier 2021. URL: http://journals.openedition.org/aam/3132 ; DOI: https://doi.org/10.4000/aam.3132

Questo documento è stato generato automaticamente il 27 janvier 2021.

\section{(c) (1) $\Theta \Theta$}

Archivio antropologico mediterraneo è distribuita con Licenza Creative Commons Attribuzione - Non commerciale - Non opere derivate 4.0 Internazionale. 


\section{Berardino Palumbo, Piegare i santi. Inchini rituali e pratiche mafiose}

Marietti, Bologna, 2020

Ferdinando Fava

NOTIZIA

BERARDINO PALUMBO, Piegare i santi. Inchini rituali e pratiche mafiose, Marietti, Bologna, 2020.

ISBN 978-8821110221 
1 Che senso dare all'omaggio che i portatori delle statue dei santi, nelle processioni cattoliche in Sicilia, nell'Italia del sud ma non solo, rendono a esponenti della criminalità organizzata e/o a loro familiari facendo appunto inchinare le statue di fronte ad essi, sostando di fronte alle loro abitazioni, lungo il tragitto e anche, se il caso, deviandolo di proposito? Cosa significano "gli inchini" per gli attori sociali che li mettono in atto e per coloro che li osservano? Queste sono le domande che Berardino Palumbo pone all'inizio del suo più recente contributo, nella cui risposta sono in gioco poste conoscitive, intellettuali e politiche notevoli. Le pagine delle cronache dei giornali e i reportage

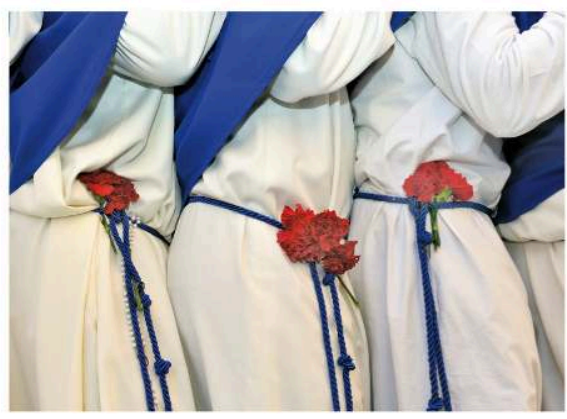
Berardino Palumbo Piegare i santi

INCHINI RITUALI E PRATICHE MAFIOSE televisivi ci hanno frequentemente presentato, secondo il ritmo stagionale delle feste religiose, molti casi di siffatti "inchini" come eventi che hanno preso la scena mediatica, tra scandalo e riprovazione, per le decisioni censorie di vescovi, parroci e forze dell'ordine coinvolti. In effetti questa visibile commistione tra devozione, religione e criminalità organizzata, così come presentata nei media sembra altamente "incommensurabile" allo sguardo di una platea di giornalisti di cronaca, di editorialisti, teologi, magistrati, scienziati sociali. "Sopravvivenze di paganesimo", con gli inchini si assiste tuttavia anche a pratiche che implicano l'esercizio di forme di violenza sui corpi dei devoti (pratiche penitenziali come la flagellazione, o atti di costrizione, come il lingere terram, ossia il leccare camminando carponi il pavimento di un edificio sacro dall'ingresso all'altare, o lo sfregare la fronte su un suo muro) o un'indebita appropriazione da parte di mafiosi, camorristi o affiliati alla 'ndrangheta, di rituali che possono al massimo essere tollerati: i tentativi di rendere conto degli inchini, appaiono a Palumbo "quasi sempre normativi, disciplinati, schematici e talvolta superficiali" (p. 12).

2 Un terreno insidioso però, non solo perché già fortemente sovradeterminato da stereotipi e interpretazioni colte che Palumbo abilmente presenta, intreccia e contestualizza nei sei capitoli di cui il testo si compone, tra una introduzione e conclusione dense, ma anche perché tali letture mainstream disegnano una geografia morale binaria nello spazio pubblico degli osservatori che definisce ideologicamente $\mathrm{i}$ confini delle risposte normativamente accettabili, confondendo comprensione e legittimazione, o come si suole dire, i giudizi di fatto e i giudizi di valore. Ebbene, Berardino Palumbo invita il lettore ad entrare con lui in questo terreno insidioso e ad attraversarlo disarticolando "fatti e valori" in relazione agli "inchini", grazie alla familiarità con i contesti e con le persone che l'intimità etnografica di vent'anni di esperienza sul campo gli conferisce, nel modus operandi che gli è proprio, quello dell'antropologo. Ed è proprio un esercizio elegante di analisi antropologica quello che egli offre in questo piccolo ma intenso testo: un gesto di ricerca e di vita, che riannoda, 
proprio interrogandosi coram populo sugli "inchini", i fili di una antropologia pubblica che intende confrontarsi con il presente e, fuori dal coro e da quelle geografie, contribuire a restituire la complessità della vita sociale, sorprendendo con spazi simbolici inattesi e aprire così ulteriori spazi di libertà all'azione individuale $\mathrm{e}$ collettiva (in tutti i modi in cui questa possa essere considerata). Il lettore è così posto sin dall'introduzione in quella posizione in-between, etnografica e epistemologica ad un tempo, che caratterizza la topologia dell'antropologo, l'assunzione dei diversi punti di vista sugli "inchini" e la decostruzione del proprio, sollecitata dallo sforzo di cogliere i primi (riconoscimento e rimessa in discussione degli assunti ideologici, dei diversi presupposti impliciti e degli atteggiamenti inconsapevolmente etnocentrici). Una posizione che sfugge alle geografie binarie con cui i saperi levigati e le economie morali dominanti hanno preteso di sezionare l'universo sociale degli attori degli inchini e fissarlo nelle frontiere delle differenze, "del paganesimo, degli anacronismi etici e culturali, o delle perverse forme di rispecchiamento tra una religiosità d'altri tempi, localistica e formale, e una socialità distorta e violenta come quella che anima le forme di vita e le pratiche di morte dei mafiosi" (p. 9). Nel solco della migliore tradizione disciplinare che non separa più il suo oggetto dal suo modo di costruirlo, Palumbo ci presenta il modus operandi utilizzato per comprendere il senso che gli attori coinvolti negli inchini attribuiscono al loro agire e gli esiti, in itinere, non scontati di tale gesto di ricerca.

3 Scarto e specchio, sono le metafore con cui illustra le condizioni di possibilità di questo progetto di conoscenza. Lo scarto dal proprio universo di pratiche e dal proprio orizzonte categoriale che si palesa nell'incontro de-centrante con questi attori e i loro universi, e che conduce alla critica genealogica e epistemologica delle proprie categorie che nell'atto del decentramento stesso sono così 'mobilizzate', "esperienza incorporata del ricercatore" (p. 7). Lo specchio che viene a costituirsi perché in questo incontro tale decentramento disloca lo sguardo dal sé del ricercatore al suo interlocutore e attraverso lo sguardo di quest'ultimo nuovamente su se stesso. Diviene così operante quella riflessività critica, epistemologica e politica (per nulla ombelicale) che proprio per l'attenzione che Palumbo pone a questo gioco di rimandi, lo autorizza non solo ad interrogare le categorie che organizzano la sua esperienza degli universi sociali della Sicilia sud orientale e l'apprensione delle forme di vita dei suoi interlocutori, la religione, la devozione, lo spazio pubblico, le pratiche violente, e i presupposti culturali relativi con cui guarda così gli inchini. Palumbo va oltre. Contestualizzando la sua posizione all'interno di più ampi scenari sociali, tra etnografia e storia, di cui egli stesso è parte, si interroga sulla genealogia del giudizio, morale, estetico, politico e culturale con cui tali inchini sono valutati. Ci mostra come il relativismo conoscitivo caratteristico del gesto antropologico, la sua vocazione originaria a porre in relazione anche dislocandoli contesti diversi, nello spazio vissuto e nella temporalità storica, fondano, di contro, in modo autentico, l'istanza etica della pratica antropologica, perché costruiscono una trama in-between di significati agiti in cui ciò che sembrava "incommensurabile" diventa intellegibile, relato, senza per altro perdere i tratti che ne fanno una forma di vita estranea all'osservatore. Detto diversamente con Waldenfels, egli riconosce, onora e restituisce l'estraneità, fa spazio a quest'altra forma di vita senza omogeneizzarla. Le domande al riguardo che Palumbo si pone mi sembrano illuminanti: "Dove si colloca e come si è costruita la cesura estetico-morale, teologico-pastorale e politico-culturale che al nostro attuale senso comune li [gli inchini $n d r$ ] fa apparire espressioni di una economia morale inaccettabile e di modi di essere e di agire 
anacronistici? [...] Quanti presupposti culturali, quante cesure epistemiche diamo per scontati - e dunque sottraiamo all'analisi - con i nostri giudizi?" (pp. 8-9). Palumbo ci porta di fatto al cuore dello sforzo del conoscere antropologico, molto spesso evitato dietro un agnosticismo "morale" metodologico sovente difensivo, ossia il confronto con il nodo dei giudizi e dei valori operanti nelle forme di vita che si vuole comprendere e nel gesto che intende comprenderle. L'assunzione consapevole delle cesure epistemiche implicate sempre in essi e il ristabilimento delle condizioni di una comprensione a misura di quel decentramento dello sguardo conducono al riconoscimento sociale delle soggettività che sempre siamo. Rispondere a simili questioni, istanza esigente per il rigore riflessivo che domanda, il controllo di un materiale etnografico esteso, di una critica storiografica erudita, e di una analisi politica lucida, è il progetto conoscitivo antropologico e del suo testo.

4 I capitoli del libro che si susseguono con un ritmo letterario attraente (e il lettore desidera conoscere lo sviluppo e la risoluzione dell'intrigo), non sono che la costruzione articolata e distinta dei diversi contesti in cui l'oggetto "inchino" viene finemente decorticato: mafiologia, spazio pubblico, devozione popolare, religione, modernità, costruzione del sé e agentività individuale nello spazio pubblico. Palumbo iscrive la sua lunga esperienza etnografica in continuità con il recente rinnovato interesse degli antropologi allo studio della mafia soprattutto in area anglofona, nella serie dei lavori fondati su prolungate esperienze etnografiche ma che, pur interessati a indagare contesti a forte presenza mafiosa e dunque a fornire letture anche di specifici ambiti criminali, non sono condotti all'interno di quei ristretti ambiti. Palumbo conosce e frequenta attori che sono stati indirettamente legati a gruppi mafiosi senza fare, con tutta evidenza, "una etnografia di cosche, 'ndrine o paranze" (p.16). Difficoltà pressoché insormontabile della mafiologia antropologica che diventa però una risorsa per comprendere il senso degli inchini. La decennale etnografia a Catalfaro (pseudonimo di un paese della Sicilia sud-orientale interna dell'area iblea) della competizione tra partiti insieme religiosi e politici diviene per Palumbo l'opportunità per dislocare lo sguardo conoscitivo su aree contigue, parallele, a quelle strettamente criminali e acquisire una conoscenza densa dei contesti sociali più ampi come una comprensione un po' più profonda dei contesti più specificamente mafiosi. Gli attori degli inchini $\mathrm{e}$ delle devozioni pubbliche, insomma, non sono necessariamente mafiosi ma dimorano in queste aree di contiguità e sono portatori anche di un ethos sociale condiviso che sa gestire i rapporti con gli appartenenti all'universo mafioso. E, viceversa, la presenza sulle macchine rituali nello spazio pubblico di figure espressamente appartenenti alla criminalità organizzata non comporta la loro appropriazione strumentale del rito o la criminalizzazione di tutti coloro che vi partecipano. È proprio a partire da quanto osservabile sulla scena pubblica delle processioni e della macchina rituale dei trionfi che Palumbo risitua l'analisi fine e dettagliata della gestione e dei gesti di questa macchina, le sue gerarchie di posizione tra baiardi, fercoli e vare, gli itinerari, le emozioni e le passioni espresse in tali gesti e in questo micro-spazio, nelle storie individuali e nella profondità di campo più ampia sulla natura di questo spazio pubblico, non riducibile a quello di una monolitica modernità. Così entriamo in casa di Zi' Mariano, e assistiamo alla sua fede e alla sua devozione alla Madonna della Stella, le cui effige segna ogni stanza della sua casa, di fronte cui versa "cento gocci di lacrime" implorando un aiuto che non tarda a manifestarsi, un miracolo ridicolizzato dal parrino a cui lo racconta. Praticante e zelante, membro della confraternita, è anche colui che, negli anni Novanta, coinvolto in un conflitto secolare tra le due parrocchie del paese, 
aveva aggredito il proprio parroco (pp. 95-102). Incontriamo anche "l'amico meccanico" di Palumbo vissuto per anni ai margini (e probabilmente, per alcuni aspetti, a disposizione) di una cosca mafiosa, legato, per tradizione familiare e intima convinzione personale, alla chiesa di San Nicolò. Anch'egli abile a sfidare il parroco e nello stesso tempo a maneggiare con delicatezza e cura la statua del Cristo morto durante le funzioni del Venerdì Santo. Veniamo a conoscere la fede di sua moglie che, rabbrividendo di fronte a un penitente che, a notte fonda, nella chiesa in cui si era recata a chiedere grazia, attraversava carponi tutta la navata leccando il pavimento, riconosce una fede e una volontà ben più forti delle sue (pp. 103-106). Ascoltando $i$ racconti della loro esperienza della fede e della devozione e, soprattutto, osservandoli in azione nella sfera politica e in quella rituale, all'interno delle quali si muovono da protagonisti, Palumbo ricostruisce le poetiche di costruzione del sé pubblico e di quello intimo di maschi "forti", restituisce interiorità alla devozione, sdoganandola dalla categoria di religiosità deviata, riconoscendo proprio sulla macchina rituale la agentività e consapevolezza ad attori che devono muoversi tra economie morali distinte in quella zona contigua dell'organizzazione mafiosa. E questo in uno spazio pubblico dove il discorso e le pratiche religiose fissano e rappresentano status sociali, gerarchie e diritti dei diversi corpi, di gruppi e singoli individui, senza che questa organizzazione dello spazio sia un masso erratico di una fase premoderna. Insomma, gli attori degli inchini sono "pienamente moderni", come Palumbo intitola l'ultimo capitolo del suo testo.

Questa sua riflessione è una occasione per neofiti e habitué della ricerca antropologica per comprendere una antropologia del presente, che negozia con soggettività tradizioni devozionali facilmente classificabili nelle survivals di tyloriana memoria. Ci interroga sulla costruzione locale dello spazio pubblico nella relazione con la creazione dello spazio sacro, seppellita oramai la universale teoria novecentesca della secolarizzazione. Ci riporta nel dibattito decennale dei religious studies sulla lived religion e la sua interpretazione. I gocci di lacrime e il lingere terram non possono più essere circoscritti nella religiosità deviata, nel residuo di paganesimo, nella superstizione, così distanti dalla fede razionale, astratta e de-corporeizzata della teologia moderna. Essi mi sembrano rinviare, molto più che a un tesoro da salvaguardare (p. 55) o educare, a quella "poetica della credenza" con cui de Certeau connotava l'antropologia del credere, necessario e paradossale cuore pulsante della istituzione religiosa. 